\title{
Sleeping Beauty-engineered CAR T cells achieve antileukemic activity without severe toxicities
}

\begin{abstract}
Chiara F. Magnani, ${ }^{1}$ Giuseppe Gaipa, ${ }^{1,2}$ Federico Lussana, ${ }^{3}$ Daniela Belotti, ${ }^{2,4}$ Giuseppe Gritti, ${ }^{3}$ Sara Napolitano, ${ }^{5}$ Giada Matera, ${ }^{1,2}$ Benedetta Cabiati, ${ }^{1,2}$ Chiara Buracchi, ${ }^{1}$ Gianmaria Borleri, ${ }^{3}$ Grazia Fazio, ${ }^{1}$ Silvia Zaninelli, ${ }^{6}$ Sarah Tettamanti, ${ }^{1}$ Stefania Cesana, ${ }^{1,2}$ Valentina Colombo, ${ }^{1,2}$ Michele Quaroni, ${ }^{1,2}$ Giovanni Cazzaniga, ${ }^{1}$ Attilio Rovelli, ${ }^{5}$ Ettore Biagi, ${ }^{1,5}$ Stefania Galimberti, ${ }^{7}$ Andrea Calabria, ${ }^{8}$ Fabrizio Benedicenti, ${ }^{8}$ Eugenio Montini, ${ }^{8}$ Silvia Ferrari, ${ }^{3}$ Martino Introna, ${ }^{3,6}$ Adriana Balduzzi, ${ }^{4,5}$ Maria Grazia Valsecchi, ${ }^{7}$ Giuseppe Dastoli, ${ }^{1}$ Alessandro Rambaldi, ${ }^{3,9}$ and Andrea Biondi, ${ }^{1,2,5}$

${ }^{1}$ Tettamanti Research Center, Department of Pediatrics, University of Milano-Bicocca/Fondazione MBBM, Monza, Italy. ${ }^{2}$ Laboratorio di Terapia Cellulare e Genica Stefano Verri, ASST-Monza, Ospedale San Gerardo, Monza, Italy. ${ }^{3}$ Hematology and Bone Marrow Transplant Unit, ASST Papa Giovanni XXIII, Bergamo, Italy. ${ }^{4}$ Department of Pediatrics, University of Milano-Bicocca, Milan, Italy. ${ }^{5}$ Clinica Pediatrica, University of Milano-Bicocca/Fondazione MBBM, Monza, Italy. 'USS Centro di Terapia Cellulare "C. Lanzani," Bergamo, Italy. 'Bicocca Bioinformatics, Biostatistics and Bioimaging Centre, Department of Medicine and Surgery, University of Milano-Bicocca, Milan, Italy. ${ }^{8}$ San Raffaele Telethon Institute for Gene Therapy (SR-TICET)/IRCCS San Raffaele Scientific Institute, Milan, Italy. ${ }^{9}$ Department of Oncology and Hematology, University of Milan, Milan, Italy.
\end{abstract}

BACKGROUND. Chimeric antigen receptor (CAR) T cell immunotherapy has resulted in complete remission (CR) and durable response in highly refractory patients. However, logistical complexity and high costs of manufacturing autologous viral products limit CAR T cell availability.

METHODS. We report the early results of a phase I/II trial in B cell acute lymphoblastic leukemia (B-ALL) patients relapsed after allogeneic hematopoietic stem cell transplantation (HSCT) using donor-derived CD19 CAR T cells generated with the Sleeping Beauty (SB) transposon and differentiated into cytokine-induced killer (CIK) cells.

RESULTS. The cellular product was produced successfully for all patients from the donor peripheral blood (PB) and consisted mostly of $\mathrm{CD3}^{+}$lymphocytes with $43 \%$ CAR expression. Four pediatric and 9 adult patients were infused with a single dose of CAR T cells. Toxicities reported were 2 grade I and 1 grade II cytokine-release syndrome (CRS) cases at the highest dose in the absence of graft-versus-host disease (GVHD), neurotoxicity, or dose-limiting toxicities. Six out of 7 patients receiving the highest doses achieved CR and CR with incomplete blood count recovery (CRi) at day 28. Five out of 6 patients in CR were also minimal residual disease negative (MRD-). Robust expansion was achieved in the majority of the patients. CAR T cells were measurable by transgene copy PCR up to 10 months. Integration site analysis showed a positive safety profile and highly polyclonal repertoire in vitro and at early time points after infusion.

CONCLUSION. SB-engineered CAR T cells expand and persist in pediatric and adult B-ALL patients relapsed after HSCT. Antileukemic activity was achieved without severe toxicities.

TRIAL REGISTRATION. ClinicalTrials.gov NCT03389035.

FUNDING. This study was supported by grants from the Fondazione AIRC per la Ricerca sul Cancro (AIRC); Cancer Research UK (CRUK); the Fundación Científica de la Asociación Española Contra el Cáncer (FC AECC); Ministero Della Salute; Fondazione Regionale per la Ricerca Biomedica (FRRB).

Authorship note: CFM and G Gaipa share first authorship. A Rambaldi and A Biondi are co-senior authors.

Conflict of interest: The Tettamanti Foundation has filed a patent application for the technology used in this report (European patent application 15801344.1; PCT/ EP02015/075980), and CFM, ST, EB, and A Biondi are inventors. The technology was licensed to Formula Pharmaceuticals for further development. Formula Pharmaceuticals provided a research grant to support the current academic study. Copyright: @ 2020, American Society for Clinical Investigation.

Submitted: April 6, 2020; Accepted: July 29, 2020; Published: October 12, 2020 Reference information: / Clin Invest. 2020;130(11):6021-6033.

https://doi.org/10.1172/JCl138473.

\section{Introduction}

Substantial efforts over the past few years have led chimeric antigen receptor (CAR) $\mathrm{T}$ cell therapy to success in relapsed and refractory $(\mathrm{r} / \mathrm{r}) \mathrm{B}$ cell malignancies. The early concept evaluated in experimental academic clinical trials moved to real-world practice, confirming the impressive clinical results (1). In $\mathrm{r} / \mathrm{r} \mathrm{B}$ cell acute lymphoblastic leukemia (B-ALL), which has chances of survival estimated at $10 \%$ to $30 \%$ at 5 years with conventional therapy (2-4), minimal residual disease-negative (MRD-) complete remission (CR) rates were observed at 1 month after CAR T cell 
infusion in $63 \%$ to $93 \%$ of the patients, with an overall survival (OS) of $60 \%$ to $80 \%$ at 6 months in multiple studies (5-10). Still, a substantial heterogeneity in clinical outcomes was observed when comparing pediatric and adult patients, different CAR T cell products, and transduction modalities (11). In addition, logistical complexity, high costs, and toxicities are currently the main barriers to the use of CAR T cell therapy. Production and handling of viral vectors are time consuming and require specialized biosafety level 2 (BSL2) facilities and trained staff resources. Furthermore, patient-derived CAR T cell production may be limited due to failure to collect sufficient $\mathrm{T}$ cells or to expand products in selected patient populations who received intensive chemotherapy. Notably, patients with a $\mathrm{T}$ cell signature predicting dysfunctional response or with high leukemic blast contamination might benefit from donor-derived products (12-14).

We therefore propose nonviral engineering of an allogeneic $\mathrm{T}$ cell population according to cytokine-induced killer (CIK) cell protocol of differentiation (15). This population is characterized by the enrichment of $\mathrm{CD}^{+} \mathrm{CD}^{2} 6^{+}$cytotoxic cells and a high profile of safety, leading to minimal occurrence of graft-versus-host disease (GVHD) after allogeneic CIK (16-18). Sleeping Beauty (SB) is an integrating vector belonging to the Tc1/mariner family of DNA transposon that was reconstructed by inverse engineering of an inactive gene in fish genome $(19,20)$. The SB transposon vector allows for permanent genetic modification through stable integration of the transgene cassette, conferring prolonged expression in $\mathrm{T}$ cells. The SB vector has recently been validated in clinical trials for the manufacture of CAR T cells (21) that were selectively propagated ex vivo with multiple stimulations in the presence of artificial antigen presenting cells (AaPCs). We have previously reported an improved platform of nonviral engineering using the $S B$ vector that consists of a single stimulation step and was applied to different CAR molecules, including anti-CD19, anti-CD123, and anti-BAFFR $(22,23)$. Herein, we report the clinical application of this concept for treating adult and pediatric patients with B-ALL relapsed after allogeneic hematopoietic stem cell transplantation (allo-HSCT) by using nonviral CD19-specific CAR CIK cells (CARCIK-CD19) manufactured from the previous transplant donor. These data demonstrate the feasibility and safety of our manufacturing platform and support further clinical application of nonviral allogeneic CAR T cell products.

\section{Results}

Product manufacturing. Nonviral SB-engineered CAR T cells were produced according to our previously established platform (24) and named CARCIK-CD19 cells. We chose to differentiate T cells to generate a memory $\mathrm{T}$ cell population according to CIK cell protocol, as the incidence of GVHD is usually less prevalent using memory cells $(17,25)$. An objective of our study was to determine the feasibility and reproducibility of manufacturing and releasing donor-derived nonviral products with a level of CAR expression higher than 20\%, an enrichment in $\mathrm{CD}^{+} \mathrm{CD}^{+} 6^{+} \mathrm{T}$ cells higher than $30 \%$, a cell viability higher than $80 \%$, in vitro potency higher than $25 \%$, and safety compliance. Cellular products were generated starting from $50 \mathrm{~mL}$ of peripheral blood (PB) from the previous transplant donor by electroporation of total PBMCs with SB plasmids expressing a CD19 third-generation CAR incorporating the CD28 and OX40 costimulatory domains (Supplemental Figure 1A; supplemental material available online with this article; https://doi.org/10.1172/JCI138473DS1). The CARCIK-CD19 batches prepared for clinical use were derived from 3 HLA identical siblings (ISD), 4 matched unrelated donors (MUD), and 6 haploidentical donors (Haplo). After electroporation, cells were differentiated by using a single stimulation with anti-CD3 and IFN- $\gamma$ and IL-2 for T cell expansion (Supplemental Figure 1B). In total, we manufactured 19 batches by seeding a median of 129.3 $\times 10^{6}$ allogeneic PBMCs. Three of these 19 batches were manufactured for the purpose of good manufacturing practices (GMP) process validation starting from $\mathrm{PB}$ of voluntary donors, whereas 16 batches were produced for clinical use from 12/20/2017 to $07 / 30 / 2019$. At the end of the expansion process, a mean of 6.91 $\times 10^{9}$ nucleated cells (range, 1.39 to $20.0 \times 10^{9}$; Figure $1 \mathrm{~A}$ ) was harvested with a mean fold increase of 71.08 (range, 14.41 to 338.8; Supplemental Figure 2A). Manufactured cells had high levels of viability (mean, $96.06 \% \pm$ SD $2.60 \%$; range, $89.10 \%-98.90 \%$ ) and were mostly $\mathrm{CD}^{+}$lymphocytes (mean, $98.92 \% \pm$ SD 1.24\%) with high killing activity and effector memory phenotype (Figure 1, B-D, and Supplemental Figure 2, B and C). Of these, a mean of $43.05 \%$ (range, $15.10 \%-73.17 \%$ ) were $\mathrm{CAR}^{+}, 46.85 \%$ (range, $31.62 \%-$ $71.80 \%$ ) were $\mathrm{CD}^{+} 6^{+}$, and $80.90 \%$ (range, $55.80 \%-93.20 \%$ ) were $\mathrm{CD}^{+}$, with a median number of transgene copies per cell of 3.54 (range, 0.62-4.92; Figure 1D and Supplemental Figure 2D). The majority of $\mathrm{CD}^{+} \mathrm{CAR}^{+} \mathrm{T}$ cells had a $\mathrm{CD} 8^{+}$effector memory phenotype (Figure 1, E and F). The median length of cell product manufacturing was 23 days (range, 20-32 days), and batches could be released 7 to 10 days after the production ended. All the enrolled patients had successful manufacturing of a CARCIK-CD19 batch (Supplemental Table 1). The quality requirements for batch release were met in 15 out of 19 productions. One batch was rejected due to a bacterial contamination. Another batch, manufactured for the same patient, was released and infused according to the original treatment schedule. Three batches partially deviated from specifications due to a lower level of CAR expression (15.01\% CD3 ${ }^{+-}$ $\mathrm{CAR}^{+}$instead of $\geq 20 \%$ ) and a higher value of transposase expression (41.68 and 97.06, respectively instead of $<20$ copies $/ 10,000$ $\beta$-glucuronidase [GUS]), but largely below the safety limit for possible enzyme activity (ref. 26 and Supplemental Figure 2E). However, the out-of-specification batches were infused (patients 6, 12 , and 13) as per clinical decision based on the absence of any alternative treatment options (27).

Clinical trial. We designed a multicentric clinical study (ClinicalTrials.gov NCT03389035) to assess the safety and feasibility of infusing allogeneic CARCIK-CD19 in patients with B-ALL relapsed after HSCT. The trial followed a 4-dose escalation scheme $\left(1 \times 10^{6}, 3 \times 10^{6}, 7.5 \times 10^{6}\right.$, and $15 \times 10^{6}$ transduced CARCIK-CD19 cells $/ \mathrm{kg}$ ) using the Bayesian optimal interval design (BOIN). From January 2018 to November 2019, a total of 20 patients were screened, and 16 were enrolled (Figure 2). Two patients were excluded from receiving lymphodepletion chemotherapy and cell infusion, one due to rapid disease progression leading to premature death and one due to acquisition of a myeloid phenotype. An additional patient decided to withdraw from the study. A total of 13 patients, 4 children and 9 adults, proceeded to lymphodepletion and treatment with a single infusion 
A

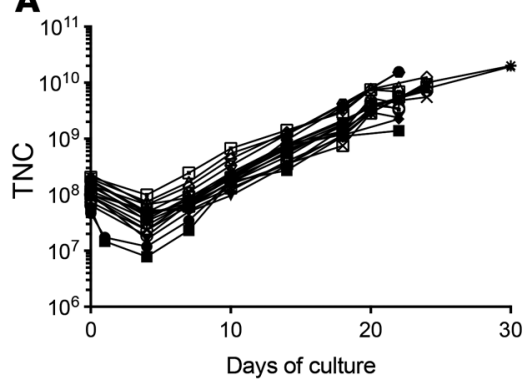

B

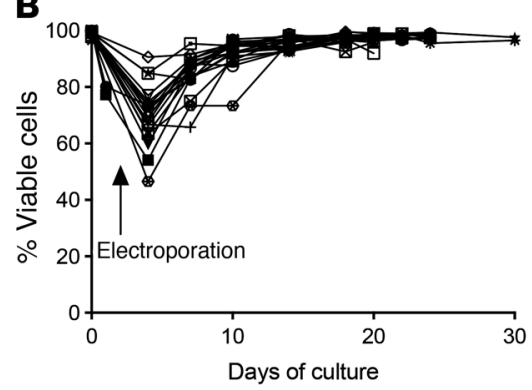

C

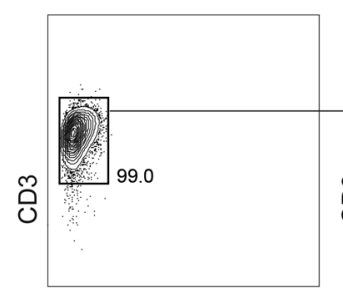

SSC

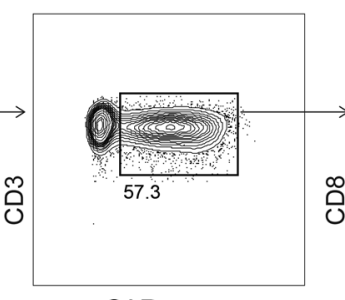

CAR

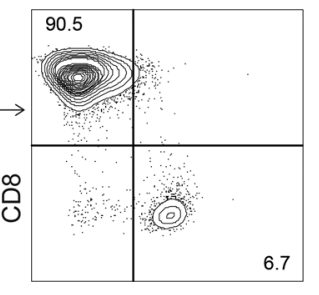

CD4

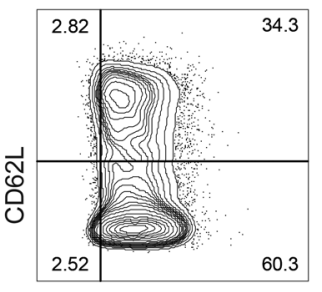

CD45RO

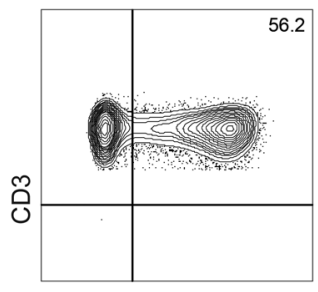

CD56
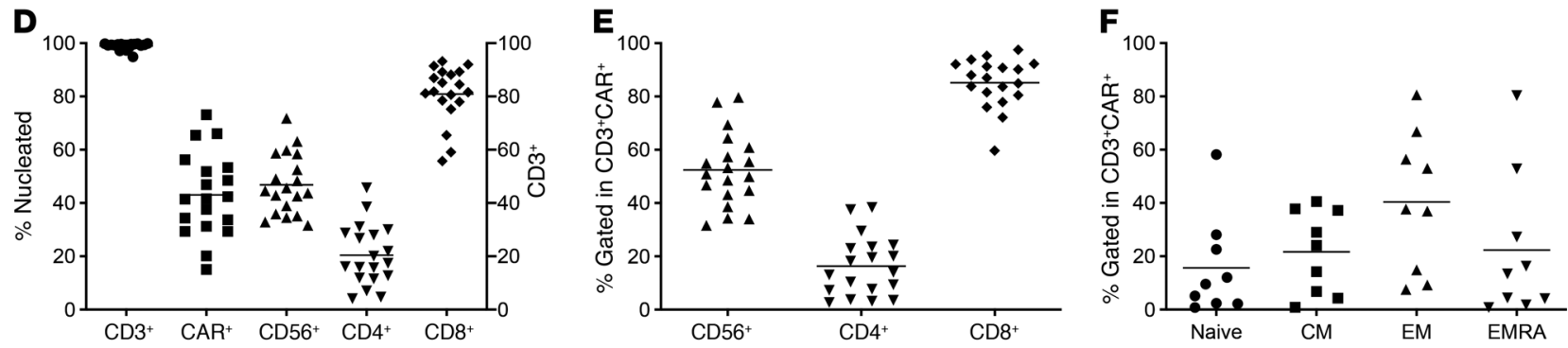

Figure 1. Cell expansion and composition of manufactured medicinal products. (A) Expansion kinetics of 19 different batches are represented as total number of nucleated cells (TNC) over time. Each line represents a single batch. (B) Viability of TNC over time $(n=19)$. Arrow indicates time point at which electroporation was performed. (C) Flow cytometric immunophenotyping by dual-density plots in 1 representative batch $(n=9)$. CD3 $3^{+}$cells were selected by CD3/side scatter (SSC) gating (left). CD3+CAR ${ }^{+}$cells were gated, and CD4/CD8, CD45R0/CD62L, and CD3/CD56 expression were measured. (D) Expression of $\mathrm{CD}^{+}, \mathrm{CAR}^{+}, \mathrm{CD}^{+} 6^{+}, \mathrm{CD}^{+}$, and $\mathrm{CD} 8^{+}$cells as percentages of TNCs. Each symbol represents a single batch. (E) Expression of CD56 ${ }^{+}, \mathrm{CD}^{+}$, and $\mathrm{CD} 8^{+}$cells as percentages of $C D 3^{+} C A R^{+} T$ cells. Each symbol represents a single batch. (F) Expression of naive, central memory (CM), effector memory (EM), and terminal effector (EMRA) cells as percentages of $\mathrm{CD}^{+} \mathrm{CAR}^{+} \mathrm{T}$ cells. Means are shown as horizontal lines.

of CARCIK-CD19 product, with a median time from enrollment to infusion of 76.6 days (range, 50-107 days). Median age was 32 years (range, 2-63 years). All patients had undergone multiple prior lines of therapy (median, 2; range, 1-7) and at least 1 allogeneic transplant, with a median of 9 months (range 2-30 months) from allo-HSCT to relapse. Seven out of 13 patients experienced acute and/or chronic GVHD after allo-HSCT and were treated with steroids (5/13), steroid and tacrolimus (1/13), or infliximab $(1 / 13)$. The BM blast count at enrollment ranged from $5 \%$ to $98 \%$, and 4 patients presented active extramedullary diseases (Table 1). Notably, the median lactate dehydrogenase (LDH), platelet, and neutrophil counts before lymphodepletion were $306 \mathrm{U} / \mathrm{L}$ (range, 148-595 U/L), 68,000 platelets/mmc (range, 12,000-237,000 platelets $/ \mathrm{mmc}$ ), and 650 neutrophils/mmc (range, 60-64,150 neutrophils $/ \mathrm{mmc}$ ), respectively, reflecting the aggressive progression of the disease that indeed required bridging therapy before infusion for all the patients (Table 1 and Supplemental Table 2).

Engraftment and expansion of CAR T cells. Detectable peripheral CAR T cell engraftment was observed in $13 / 13$ patients (100\%) by transgene copy quantitative evaluation and in $12 / 13$ patients (92.9\%) by flow cytometry using a recombinant human CD19 pro- tein, with robust expansion $(>100,000$ copies per $\mu \mathrm{g}$ DNA and $>40$ cells per $\mu \mathrm{l}$ ) achieved in $8 / 13$ patients (Figure $3, \mathrm{~A}-\mathrm{C}$ ). The median time to maximal expansion was 14 days (range, 7-22 days), and the maximal expansion reached about $1 \times 10^{6}$ transgene copies per $\mu \mathrm{g}$ DNA and $70 \%$ of CAR ${ }^{+} \mathrm{T}$ cells in PB with a median AUC from day 0 to day 28 of $1.08 \times 10^{6}$ copies per $\mu \mathrm{g}$ DNA (range, 3,915.5-4.80 $\times$ $10^{6}$; Supplemental Table 3). Engrafted CAR T cells were detected also in BM (Supplemental Figure 3, A and B). CAR T cells were effective in promoting ablation of malignant $\mathrm{CD} 19^{+}$cells in $\mathrm{PB}$ and BM. $\mathrm{CD} 19^{+}$cell ablation was achieved in association with expansion and persistence of CAR T cells, even in a patient with massive BM blast infiltration persisting after lymphodepletion (Figure 3D). CAR T cell expansion was influenced by the dose level in terms of exposure (median AUC-28d, where 28d indicates measurement from time 0 to $28,6.0 \times 10^{5}, 5.3 \times 10^{4}, 1.3 \times 10^{6}$ and $2.2 \times 10^{6}$ for patients treated with dose levels $1,2,3$, and 4, respectively) and time to reach expansion (Figure 3, E-G) as well as by the tumor burden after lymphodepletion (median AUC-28d, $3.4 \times 10^{5}$ and 2.7 $\times 10^{6} ; \mathrm{C}_{\max }-28 \mathrm{~d}, 4.2 \times 10^{4}$ and $3.8 \times 10^{5}$ for patients with less than or greater than $15 \%$ tumor burden, respectively). Interestingly, expansion was similar in pediatric and adult patients and did not 


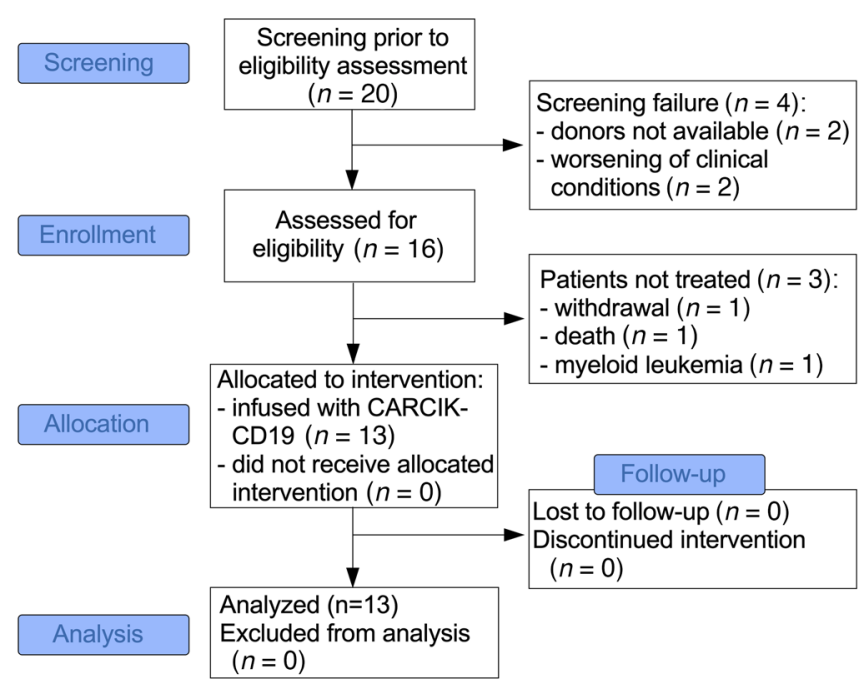

Figure 2. Study flow. Study participant flow chart from the time of screening to treatment.

depend on the type of donor (Supplemental Figure 3, C-F). CD8 ${ }^{+}$ $\mathrm{T}$ cells represented the predominant circulating $\mathrm{CAR}^{+} \mathrm{T}$ cell subset along with $\mathrm{CD}^{+} \mathrm{CD}^{+} 6^{+} \mathrm{CIK}$ cells and $\mathrm{CD} 4^{+} \mathrm{T}$ cells to a lesser extent. Persistence of central memory $\mathrm{CAR}^{+} \mathrm{T}$ cells was observed after infusion. The majority of CAR T cells had a central and effector memory phenotype in vivo (Figure 4, A-C). Twelve out of 13 patients had detectable CAR T cells measured as transgene copy number at the last available assessment, with a median duration of 94 days (range, 22 to 300 days).

Toxicity. Toxicities are summarized in Table 2 and Supplemental Table 4. The infusion was uneventful in all patients except 1 pediatric patient (patient 6), who experienced generalized seizures, tachycardia, and loss of consciousness due to a DMSO reaction, which rapidly responded to diazepam. No further infusion-related reactions were observed. Two grade I and 1 grade II cytokine-release syndrome (CRS) cases (assessed with criteria in Lee et al. [ref. 28]; Supplemental Table 4) occurred in 3 patients treated with the highest dose. CRS was associated with increased concentrations of serum cytokines (Supplemental Figure 4). Patient 12, who was the only patient experiencing grade II CRS, with fever and hypoxia, required low-flow oxygen $\left(\mathrm{FiO}_{2}\right.$ $<40 \%$ ) and treatment with 2 doses of tocilizumab with benefit and resolution of symptoms. The day of the infusion, patient 5 experienced pneumonitis with acute respiratory distress, which required oxygen therapy and antibiotics; tocilizumab was also infused at day +1 after infusion, as CRS could not be excluded at the time. On day +10 , for a worsening of respiratory failure, the patient was transferred to an intensive care unit (ICU), where she received noninvasive ventilation. Clinical improvement followed methylprednisolone and further antibiotic changes. Deep pancytopenia was reported in 2 out of 13 patients and severe infections in 4 patients. Consistent with the concomitant in vivo detection of CAR T cells, B cell aplasia (BCA) was observed in all treated patients. Six of these patients had persistent BCA at the last follow-up, with a median duration of 3 months (Figure 5A). None of the treated patients developed immune-effector cell neuro- toxicity syndrome (ICANS). Furthermore, the persistent engraftment of allogeneic CAR T cells was not associated with any sign or symptom of GVHD, although some patients had experienced GVHD after previous allo-transplant.

Response data. The overall response rate (CR/CR with incomplete blood count recovery [CRi]) was $61.5 \%$ (8 out of 13 patients, $95 \% \mathrm{CI}=31.6 \%-86.1 \%)$. Six out of 7 patients, receiving the 2 highest doses, achieved CR/CRi, with an overall response rate at day 28 after infusion of $85.7 \%$ ( $95 \% \mathrm{CI}=42.1 \%-99.6 \%$, Figure $5 \mathrm{~B}$ and Table 2). Patient 6 experienced BM remission, but persistent extramedullary disease with a mixed $\mathrm{CD}_{19}{ }^{+} \mathrm{CD} 19^{-}$phenotype and was not accounted as a responder, accordingly. MRD was assessed by both multiparametric flow cytometry and PCR for leukemia-specific immunoglobulin (IG)/T cell receptor (TR) gene rearrangements in all responder patients. Five out of the 6 patients $(83.3 \%, 95 \% \mathrm{CI}=35.9 \%-99.6 \%)$ in $\mathrm{CR}$ at the highest doses were also $\mathrm{MRD}^{-}$. $\mathrm{MRD}^{-}$status was achieved by $75 \%$ of all responders ( 6 out of $8,95 \% \mathrm{CI}=34.9 \%-96.8 \%$ ). The 2 patients in $\mathrm{CR}$, but who were $\mathrm{MRD}^{+}$, relapsed with a $\mathrm{CD} 19^{+}$disease at +2.3 and +1.9 months after infusion, respectively, and one of them is alive and receiving salvage therapy. Among the 6 patients who achieved $\mathrm{MRD}^{-} \mathrm{CR}, 2$ children underwent consolidation with a second allo-HSCT and are still alive and disease free $(+14$ and +10 months), 2 adult patients died of subsequent $\mathrm{CD} 19^{+}$disease relapse, and 2 adult patients who did not undergo allo-HSCT are still alive and disease free ( +12 and +9 months) without additional therapies at +9 months from the data cutoff date. Interestingly, patient 12 relapsed after allo-HSCT with a diffuse B-ALL presenting elevated S-aspartate aminotransferase (AST)/S-alanine aminotransferase (ALT) $(157 / 287 \mathrm{UI} / \mathrm{L})$, S- $\gamma$ glutamil transpetidase $(\gamma \mathrm{GT})(1,183$ $\mathrm{UI} / \mathrm{L})$, and bilirubin (Bil) $(18.8 \mathrm{mg} / \mathrm{dL})$ due to malignant liver infiltration. CT scan performed 44 days after treatment showed shrinking of the tumor mass associated with recovery of liver function (AST/ALT, 12/58 UI/L; $\gamma \mathrm{GT}: 82 \mathrm{UI} / \mathrm{L} ; \mathrm{Bil}, 0.8 \mathrm{mg} / \mathrm{dL}$ ) (Figure 5C). This latter case indicated the ability of CIK cells to infiltrate large tumor masses (29) and to tackle extramedullary leukemia. Such ability was further corroborated by the detection of massive infiltration of CAR T cells in the $2 \mathrm{~L}$ pleural effusion. The overall median follow-up among the patients who received CARCIK-CD19 infusion was 8.7 months (range, 1.02-20.03 months). So far, 4 of the 7 patients treated with the last 2 highest dose levels are alive and in CR with a median follow-up of 6.9 months as of the data cutoff date, whereas 3 adult patients relapsed, all with retention of CD19 antigen expression.

Integration site analysis. We performed a comprehensive integration site (IS) analysis on genomic DNA extracted from the medicinal product batches at release and from the PB of 13 treated patients, harvested at different time points (ranging from 7 to 28 days) after infusion. Moreover, we also analyzed a BM sample from a single patient (strategy depicted in Figure 6A). For the retrieval of SB IS, we adopted a technique similar to linker-mediated (LM) PCR (30), where genomic DNA is sheared by sonication, ligated to a DNA linker cassette, and amplified by PCR using oligonucleotides complementary to the vector sequence and the linker cassette bound to the shear site and then sequenced (NCBI's Sequence Read Archive [SRA] PRJNA643365). On average, CARCIK-CD19 batches yielded a higher amount of PCR products compared with in 


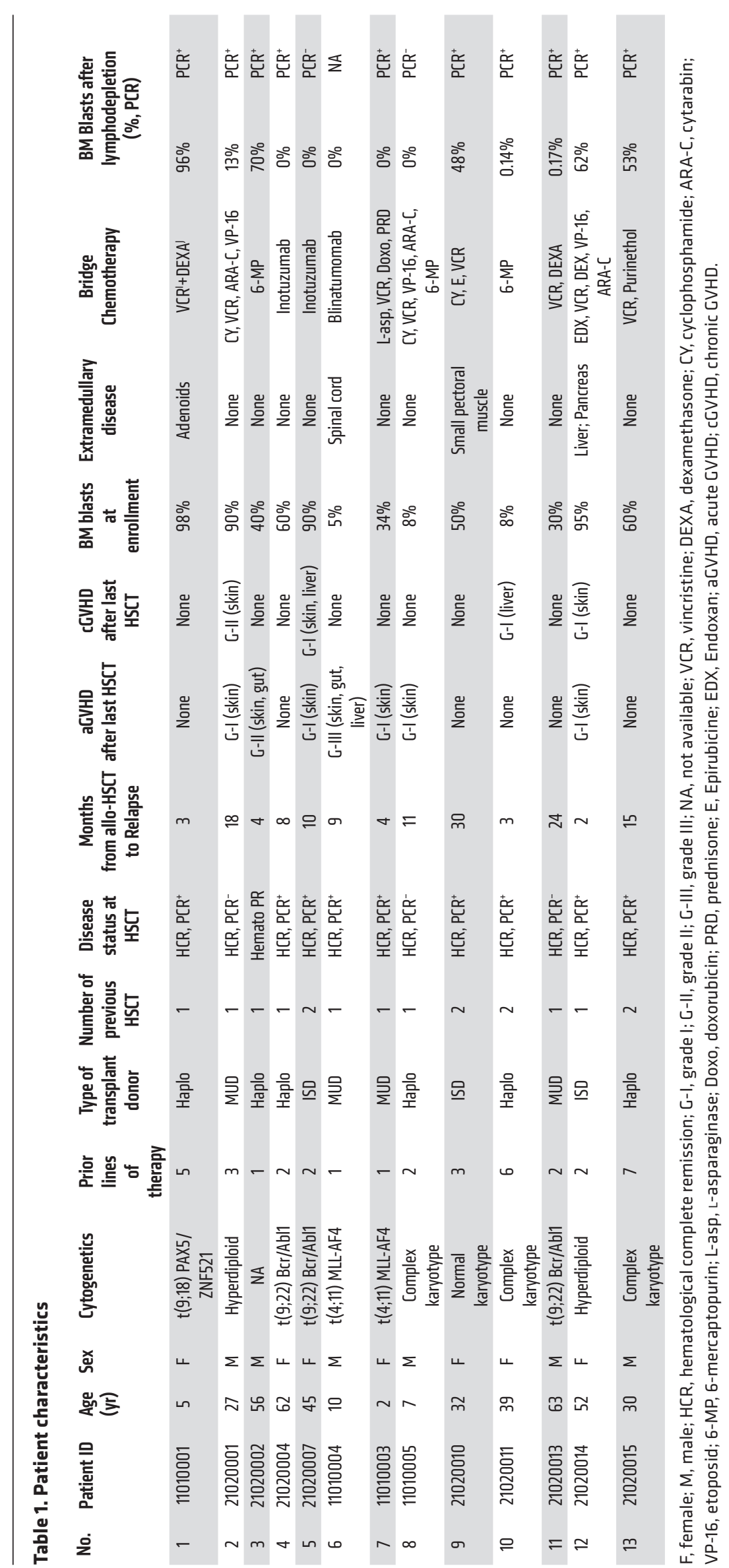

vivo samples (Supplemental Figure 5). By this approach, we identified a total of 101,020 and 88,034 IS in in vitro and in vivo samples, respectively (Supplemental Table 5). The relative number of retrieved IS for each sample was proportional to the marking levels (Supplemental Figure 6). The distribution of SB IS around the transcription start site (TSS) showed no bias for integratations near promoter sequences (Figure 6B). Gene ontology enrichment analysis, performed with the Genomic Regions Enrichment of Annotations Tool (GREAT), revealed a significant enrichment of genes involved in T cell biology including $\mathrm{T}$ cell activation, differentiation, and proliferation (Supplemental Figure 7). To identify additional signs of insertional mutagenesis, we searched for common insertion sites (CIS). We identified sporadic CIS in single patients and targeting nearby genes by a relatively small number of IS (3 to 5), and none of them was classified as a cancerrelated gene (Figure 6C and Supplemental Figure 8). All in vitro and in vivo samples harvested at early time points showed a highly polyclonal repertoire, reaching thousands of distinct IS in several samples, with the greatest represented IS in terms of abundance of $4 \%$. All patients showed identical IS persisting over different time points, although no clonal expansions were observed in any patient. However, at later time points $(\geq$ 28 days after infusion), the repertoire of IS showed a marked reduction toward oligoclonality, which was further evidenced by the analysis of the Shannon diversity index (Figure 6, D and E, and Supplemental Figure 9). The increase in clonal abundance in the late data sets was similar among all IS and was not enriched for IS targeting cancer-related genes, suggesting that the observed oligoclonality was not caused by the appearance of specific dominant clones, but was rather the result of the reduction in the number of retrieved IS.

\section{Discussion}

This study provides the first evidence, to our knowledge, that donor-derived cells engineered with the SB transposon are a safe and valid therapeutic option for B-ALL patients relapsed after allo-HSCT. This platform reproducibly produces CARCIK-CD19 cellular products starting from a small amount of donor-derived PB. Manufactured cells were able to expand rapidly and efficiently in vivo, with persistence measurable for up 
A

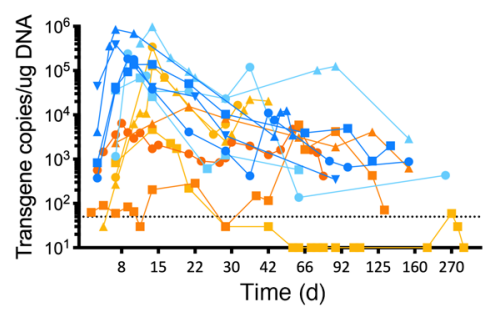

B

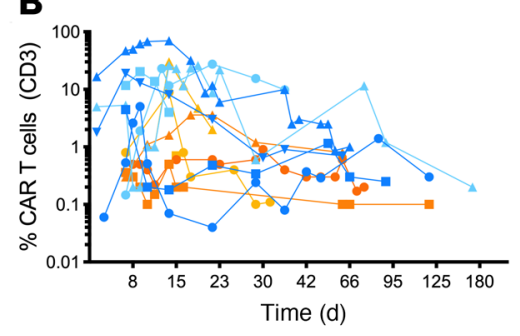

C

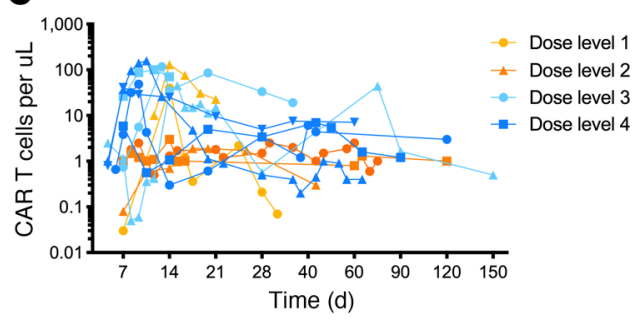

D
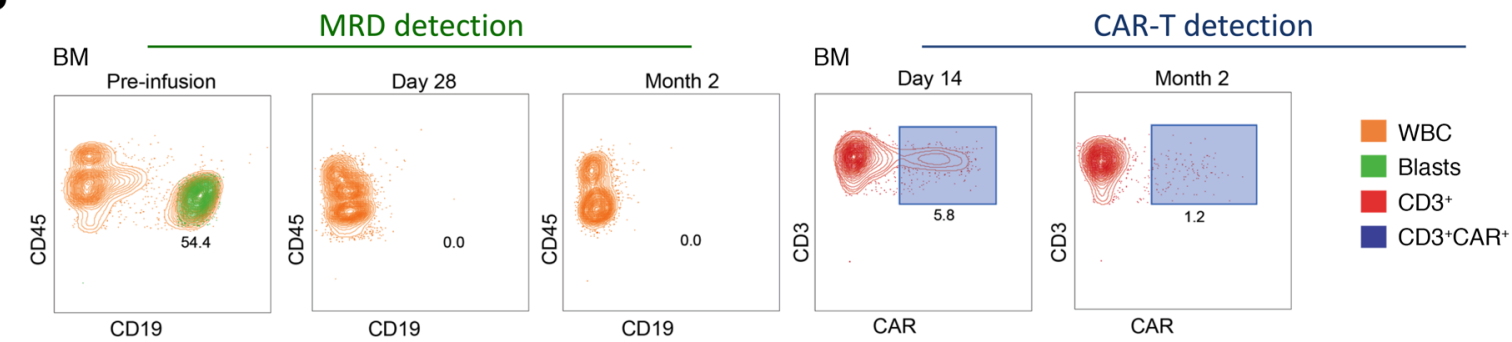

PB
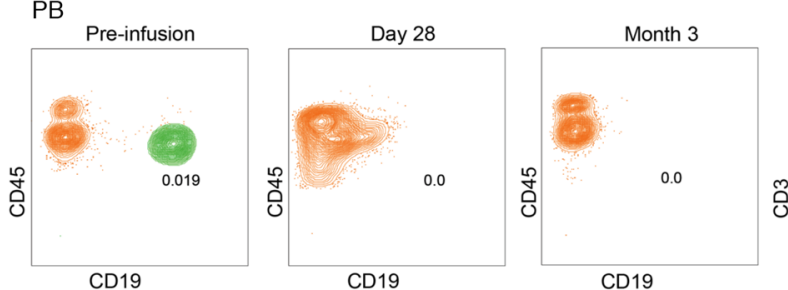

PB
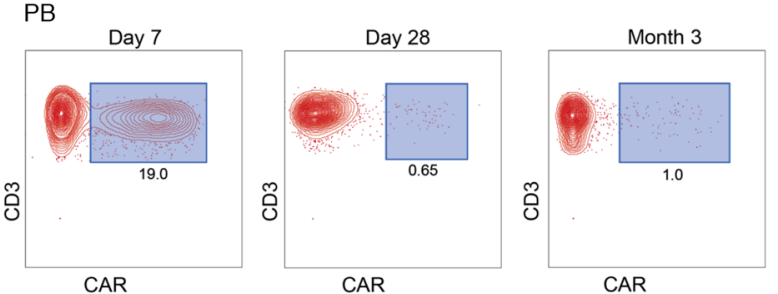

E

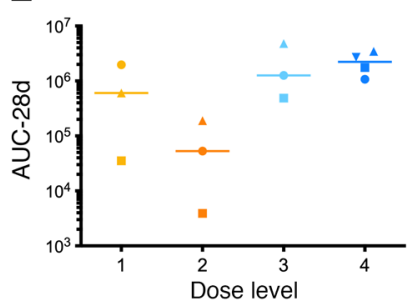

$\mathbf{F}$

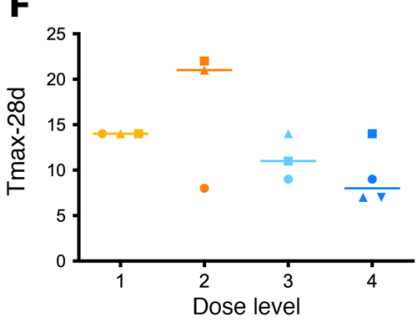

G

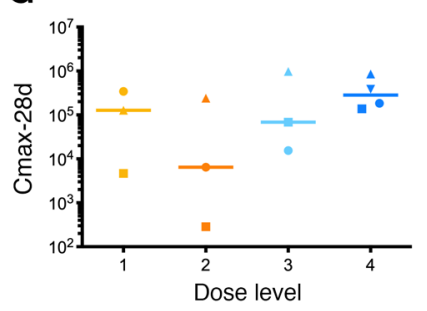

Figure 3. Postinfusion expansion and persistence of CAR T cells in PB according to dose level. Transgene copy number per $\mu$ in blood (A), percentage of $C A R^{+} T$ cells within the total $C D 3^{+} T$ cells in blood (B), absolute counts of $C A R^{+} C D 3^{+}$cells in blood (C) measured at different intervals of time after CARCIK-CD19 infusion in patients treated at different dose levels. Each symbol and color codifes an individual patient sample ( $n=13)$. Measures under LOQ $(<50$ copies $/ \mu \mathrm{g}$ ) were inserted in the graphs with a fixed reference value. (D) Flow cytometric dual-density plots showing leukemic blast clearance assessed as MRD detection and CAR T cell engraftment in BM (upper panels) and PB (bottom panels) at different time points in patient 13. Numbers within the diagrams represent the percentages of cells. (E) AUC-28d according to dose level. (F) $T_{\max }-28 \mathrm{~d}$ according to dose level. (C) $C_{\max }-28 d$ according to dose level. Each symbol represents a single patient $(n=13)$. AUC (transgene copies/ $\mu \mathrm{g}$ DNA).

to 10 months in patient's blood and BM. The infusion of CARCIK-CD19 was remarkably safe in all treated patients, since CRS incidence and grade were negligible and acute GVHD and neurotoxicity were never observed. At the higher dose levels, a substantial rate of hematological and molecular response was achieved. Most of the responders are still in remission at this writing, with an average follow-up of 6 months. Therefore, early results from our trial suggest that allogeneic nonviral manufacturing is a valid strategy for generating CAR-mediated immunotherapy.

So far, manufacture of CAR T cells, including the existing FDAand European Medicines Agency-approved (EMA-approved) products, has relied on viral vectors, which take a minimum of 2 weeks for production and require multiple steps and quality assessments $(5,31-33)$, leading to costs as high as about $30 \%$ of the cost of the entire process and difficulties in securing a viral supply in due time. In our study, we showed that $50 \mathrm{~mL}$ of blood was more than adequate for manufacturing sufficient numbers of CARCIK-CD19 for 13/13 patients. The process has a relatively low cost of goods, with an estimated cost ranging from 5 to 10 times lower than viral processes, with an average transduction efficiency and final cell viability comparable to that of viral vectors. A potential weakness of our study is represented by the duration of in vitro culture currently required to generate CARCIK-CD19 cells, mainly due to the mandatory complete clearance of SB11 transposase activity. SB11 transposase-expressing plasmid, though bearing the transiently active cytomegalovirus early promoter, causes at low frequency extended transposase protein expression that could potentially lead to remobilization of the transposon in other genomic compartments (34). For a further platform implementation, the hyperactive SB100X (35) variant in mRNA (36) or 
A

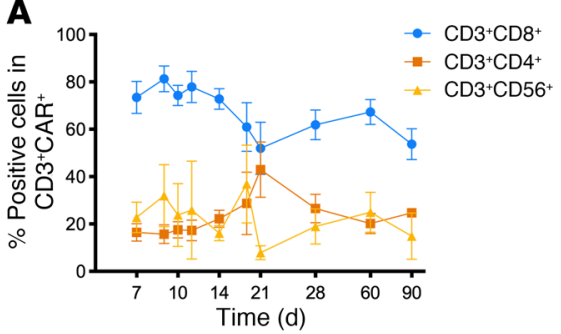

B

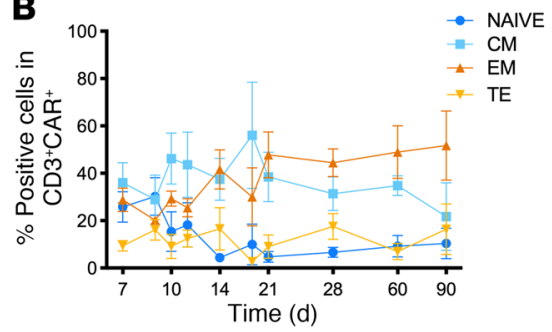

C
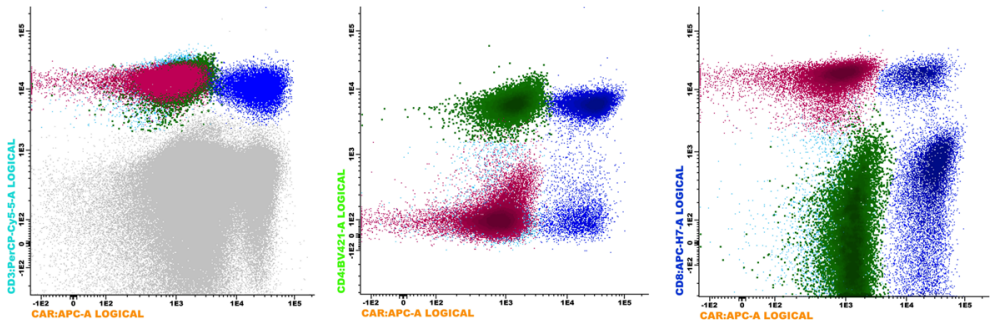

$\mathrm{CD}^{+} \mathrm{T}$ cells

$\mathrm{CD}^{+} \mathrm{T}$ cells

CAR T cells

Figure 4. Postinfusion immunophenotype and kinetic of CAR T cells. (A) Percentages of $\mathrm{CD3}^{+} \mathrm{CD}^{+}, \mathrm{CD3}^{+} \mathrm{CD}^{+}$, and $\mathrm{CD3}^{+} \mathrm{CD}^{+} 6^{+}$cell subsets within the $\mathrm{CAR}^{+} \mathrm{CD}^{+} \mathrm{T}$ cells in $\mathrm{PB}$ at different intervals of time after CARCIK-CD19 infusion $(n=11)$. (B) Expression of naive, central memory, effector memory, and terminal effector cells as percentages of $C A R^{+} C D 3^{+} T$ cells in PB at different intervals of time after CARCIK-CD19 infusion $(n=11)$. (C) Flow cytometric dot plots showing the phenotype of circulating CAR T cells in a representative B-ALL patient at 21 days after CARCIK-CD19 infusion $(n=13)$.

protein (37) version could be used. These alternatives may lead to a level of integration of SB minicircles up to $45 \%$ and $20 \%-30 \%$, respectively, thus avoiding the theoretical risk of chromosomal integration and reducing the time of in vitro culturing.

The rationale of using donor-derived memory T cells as CIK cells over conventional CAR T cells comes from clinical experiences clearly demonstrating safety and tolerability of repeated CIK cell infusions with minimal and manageable GVHD occurrence $(17,18,38)$. Minimal GVHD results from acquisition of an NK-like MHC-unrestricted cytotoxity during stimulation with $\mathrm{CD} 3$ and IFN- $\gamma$ and differentiation in the presence of IL-2 (29). In our study, we observed no occurrence of GVHD even in the context of patients who had undergone haplo-HSCT and accordingly received CARCIK-CD19 cells from their haploidentical donor. Interestingly, even patients who experienced GVHD after the initial HSCT did not show any signs of GVHD after use of CARCIK-CD19 produced from the PB of the same allogeneic donor. The absence of GVHD occurrence was consistent with recent clinical studies with donor-derived memory $\mathrm{T}$ cells after allo-HSCT (25). The small amount of starting material and the lack of GVHD activity pave the way for the development of an off-the-shelf product (39). In keeping with our strategy, other studies are currently exploiting the use of unrelated donorderived TCR-edited T cells (40-43) or cord blood-derived NK cells $(44,45)$. However, with respect to NK, T cells have a sustained in vivo persistence, which we have also reported for CARCIK-CD19, while that persistence is difficult to achieve with nonengineered NK cells (45). Genome editing is rather limited by enrichment requirements and risk of translocations and rearrangements. Similarly to our platform, differentiation

of HSC using a CD28-based CAR construct drove progeny differentiation to an NK-like phenotype (46) associated with antigendirected antileukemic activity across MHC barriers (47).

SB-engineered $\mathrm{T}$ cells used as adjuvant therapy after transplantation and administered without additional lymphodepletion showed limited expansion and persistence, with an average of 201 and 51 days for autologous and allogeneic recipients, respectively, in the absence of BCA (21). In our study, we found excellent expansion of infused CAR T cells with a cumulative exposure of $1.08 \times 10^{6}$ copies per $\mu \mathrm{g}$ DNA, as assessed by the median AUC, and a median time to maximal expansion of 14 days, which was comparable to that reported in previous studies in pediatric and adult patients treated with second-generation viral CD19CARs (10, 48). Likewise, expansion was associated with clinical activity and led to BCA. Interestingly, patients with higher tumor burden persisting after lymphodepletion experienced higher exposure and reached maximal expansion faster, which further corroborates the observation that antigen-driven stimulation drives CAR T cell expansion (12). While it has been clearly demonstrated that tumor burden is an important factor contributing to CAR T cell expansion, the impact of the infused cell dose remains controversial (7, 49). An association between cell dose and CAR T cell expansion was observed, which may be determined by the prevalence of effector memory phenotype in the cell product. The most effective treatment doses were higher than the $\mathrm{T}$ cell dose previously reported for other CAR T cell products. It is likely that the predominance of $\mathrm{CD} 8^{+}$effector memory cells, the costimulatory design, and the use of SB transposon require the infusion of higher doses of CARCIK-CD19. For the same reasons, these cells are associated with a better safety profile characterized by the absence of massive cytokine secretion. Persistent engraftment was detected at low levels up to 300 days and in the vast majority of patients at the last available measure. The use of standard lymphodepletion and the incorporation of the OX40 module in the CD28-based CAR (50) might have contributed to long-term CAR T cell persistence, as observed with a combination of costimulatory domains in some third-generation CAR structures $(51,52)$. In association with CAR T cell massive expansion, marked cytokine increase in the serum levels and severe toxicities have occurred in most CAR T clinical trials $(6-9,49)$. The modulation of the typical cytokine production storm, associated with aggravation of CRS mediated by IFN- $\gamma$, IL-6, TNF- $\alpha$, and IL-8, might explain the lower incidence of CRS and the absence of neurotoxicity despite an expansion equivalent to that in other CAR trials (53).

We found for the first time, to our knowledge, that the integration profile of SB, without preferences for gene dense regions and with no bias to target regions near TSS, is maintained after infusion, suggesting a lack of selective pressure for genomic 
Table 2. Toxicity and response to therapy

\begin{tabular}{|c|c|c|c|c|c|c|c|c|c|c|}
\hline No. & Patient ID & $\begin{array}{c}\text { Dose } \\
\left(10^{6} / \mathrm{Kg}\right)\end{array}$ & GVHD & CRS & Neurotoxicity & $\begin{array}{l}\text { Disease } \\
\text { response } \\
\text { (day 28) }\end{array}$ & $\begin{array}{c}\text { MRD qPCR } \\
\text { BM (day 28) }\end{array}$ & $\begin{array}{l}\text { Duration of } \\
\text { response (months } \\
\text { from CR) }\end{array}$ & $\begin{array}{l}\text { Overall survival } \\
\text { from infusion } \\
\text { (months) }\end{array}$ & $\begin{array}{c}\text { Current } \\
\text { status }\end{array}$ \\
\hline 1 & 11010001 & 1 & None & None & None & NR & Positive & - & 1.0 & Dead \\
\hline 2 & 21020001 & 1 & None & None & None & NR & Positive & - & $20.0^{c}$ & Alive \\
\hline 3 & 21020002 & 1 & None & None & None & NR & Positive & - & 1.6 & Dead \\
\hline 4 & 21020004 & 3 & None & None & None & CR & Positive & 1.4 & 4.0 & Dead \\
\hline 5 & 21020007 & 3 & None & None & None & CR & Negative & 3.9 & 9.8 & Dead \\
\hline 6 & 11010004 & 3 & None & None & None & NR & Positive & - & 6.1 & Dead \\
\hline 7 & 11010003 & 7.5 & None & None & None & $\mathrm{CR}$ & Negative & $8.7^{B}$ & $9.6^{0}$ & Alive \\
\hline 8 & 11010005 & 7.5 & None & None & None & $\mathrm{CR}$ & Negative & $7.8^{\mathrm{B}}$ & $8.7^{0}$ & Alive \\
\hline 9 & 21020010 & 7.5 & None & None & None & NR & Positive & - & $7.8^{E}$ & Alive \\
\hline 10 & 21020011 & 15 & None & Grade 1 & None & CR & Positive & 1.0 & 6.1 & Alive \\
\hline 11 & 21020013 & 15 & None & None & None & CR & Negative & $4.6^{B}$ & 5.4 & Alive \\
\hline 12 & 21020014 & 15 & None & Grade 2 & None & CRi & Negative & 1.1 & 2.2 & Dead \\
\hline 13 & 21020015 & 15 & None & Grade 1 & None & CR & Negative & $2.2^{\mathrm{B}}$ & 3.1 & Alive \\
\hline
\end{tabular}

NR, no response. ${ }^{A} C R$ in BM with extramedullary disease. ${ }^{B}$ Ongoing response. ${ }^{C}$ Patient 2 received inotuzumab (0.83 months from infusion) and, subsequently, HSCT. ${ }^{D}$ Patients 7 and 8 underwent HSCT (2.08 and 5.32 mos from infusion, respectively). EPatient 9 received inotuzumab ( 3.74 months from infusion)

regions with different properties in vivo. Moreover, gene ontology analysis showed a significant enrichment of genes expressed in $\mathrm{T}$ cells, in agreement with the typical SB profile characterized by a bias for targeting accessible chromatin regions that, in this case, are $\mathrm{T}$ cell specific, possibly due to the temporal proximity of integration with the CD3-specific activation $(22,54)$. As shown previously, viral insertions in HIV-infected subjects may alter $\mathrm{T}$ cell regulatory pathways and thus promote clonal expansion and, consequently, persistence of the latent HIV reservoir (55). Similarly, vector insertions in CAR T cells might trigger clonal expansions of genetically modified cells, showing dramatic in vivo expansion of a single clone. As documented in CAR T cell trials using viral vectors, vector insertion within the $C B L$ oncogene and disruption of the normal TET 2 allele were reported $(56,57)$. Despite a recent study suggesting that vector insertions might promote CAR T clonal expansion, which correlates with positive outcome (58), we did not find any evidence that SB insertions in our trial have triggered any clonal dominance nor selection of genes targeted by CIS that have been associated with potential leukemogenic insertional events in preclinical and clinical gene therapy. Importantly, a sizable amount of IS present in CARCIK-CD19 was also found in vivo, indicating that the progeny of infused CAR T cells were able to engraft, expand, and persist over time. At later time points, the clonal repertoire was markedly reduced, in agreement with the observed contraction phase, as measured by transgene PCR and flow cytometry.

We observed a dose-dependent clinical response associated with low toxicity in a category of patients with $\mathrm{r} / \mathrm{r}$ B-ALL following allo-HSCT (59), whose chances of survival are very low. The infusion of high CARCIK-CD19 doses induced CR/CRi in 6 out of 7 patients, 4 of whom remained in remission with a median follow-up of 6.9 months as of the data cutoff date. BCA was sustained at the last follow-up in 6 out of 13 patients, with a median duration of 3 months. The occurrence of CD19- relapses was limited, in keeping with that reported in other adult patient series $(7,8)$. The limited number of pediatric patients treated so far prevents us from drawing premature statements in this setting. Overall, we are tempted to speculate that CARCIK-CD19 may exert an antileukemic effect that is not limited to the CAR engagement, but may involve long-lasting allogeneic recognition. Furthermore, the predominance of the effector memory $\mathrm{T}$ cell phenotype, the large number of produced cells, and their safety profile will allow us to design subsequent studies with multiple maintenance infusions. Despite the data looking very encouraging in terms of safety and efficacy, the small number of treated patients makes it difficult to claim that this approach is as effective as commercially available CAR T cell products. Likewise, assessment of the CARCIK-CD19 production variability in relation to the treatment outcome is not possible at this time. Interestingly, the lack of GVHD activity when using HLA-mismatched donors and our ability to generate cord blood-derived CIK cells (39) open the possibility of using cord blood-derived CARCIK-CD19 even in patients who did not undergo HSCT. Following a deep lymphodepletion aimed at preventing rejection and allowing robust in vivo expansion, this approach may mostly benefit patients who fail collection or manufacturing of autologous CAR T cells.

\section{Methods}

Manufacturing. CIK cells were generated from $50 \mathrm{~mL}$ of donorderived $\mathrm{PB}$ by electroporation with GMP-grade CD19.CAR/ pTMNDU3 and pCMV-SB11 plasmids (24) (manufactured by VGXI Inc.) according to the method described in European patent EP20140192371 (60). Full details of the manufacture and release are provided in the Supplemental Methods. Briefly, PBMCs were electroporated using the 4D-Nucleofector System (Lonza) with the P3 Primary Cell 4D-Nucleofector Kit (Lonza) in the presence of a $\mathrm{pT}$ $\mathrm{SB}$ vector expressing the FMC63-derived CD19-specific scFv fused to a CD28 transmembrane domain and the CD28.OX40.CD3 3 sig- 
A

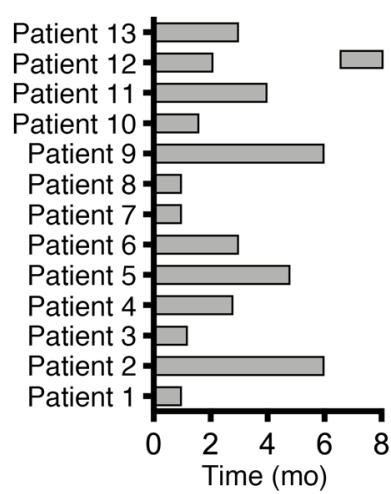

C

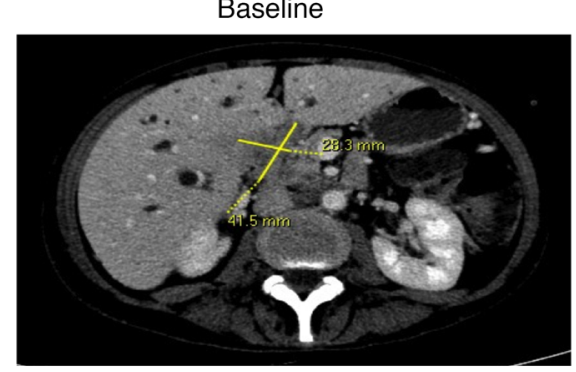

B

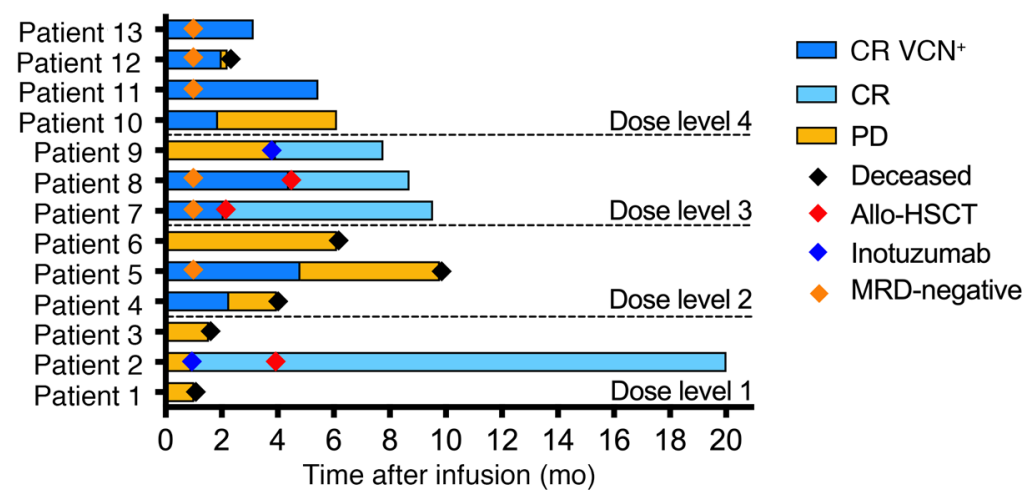

Day + 44 after CARCIK-CD19 infusion

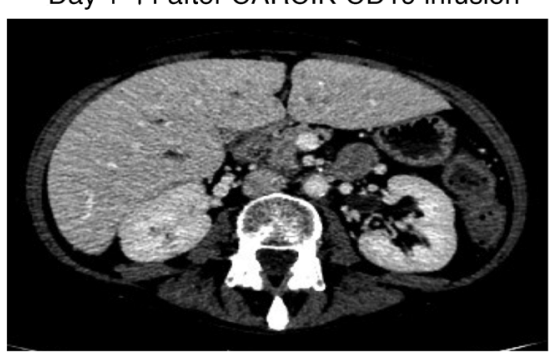

Figure 5. Clinical outcome and antileukemic response duration. (A) Waterfall plot of individual patient BCA duration. (B) Waterfall plot of individual patient remission duration $(n=13)$, remission duration in presence of transgene copy number, and timing of relapse, allo-HSCT, and eventually death. (C) CT scan at baseline and 44 days after CARCIK-CD19 infusion in patient 12 with a diffuse B-ALL presenting with massive liver infiltration. VCN, vector copy number; $\mathrm{PD}$, progressive disease.

naling endodomain under the control of the synthetic MNDU3 (61) promoter and flanked by $\mathrm{pT}$ inverted repeats (IR)/direct repeats (DR) sequences (20) and the PCMV vector encoding the transposase SB11 gene (62). Autologous PBMCs irradiated with $60 \mathrm{~Gy}$ of $137 \mathrm{Cs} \gamma$-rays were added after electroporation. IFN- $\gamma(1000 \mathrm{U} / \mathrm{mL}$; Boehringer Ingelheim) was added at day 0 , and IL-2 (300 U/mL; Novartis) and OKT-3 (50 ng/mL; Takara) were added at day 1 . Cells were then cultured in GMP Advanced RPMI-1640 Medium (Gibco, Thermo Fisher Scientific) supplemented with heat-inactivated GMP FBS (Hyclone, GE Healthcare) for 18 to 28 days, and IL-2 was added weekly. CARCIK-CD19 were formulated with cryopreservation $(23.9 \%$ of normal saline solution for intravenous injection; $56.1 \%$ of HSA solution, at $20 \%, 10 \%$ of DMSO, and $10 \%$ of ACD-A).

Study design. The study was conducted at the Pediatric Clinic of the University of Milano-Bicocca/Fondazione MBBM for 1- to 17-yearold patients and at the Papa Giovanni XXIII Hospital for patients older than 18 years. The target population consisted of patients with B-ALL who were either refractory or relapsed after allo-HSCT. Here, we report the early results on the first 13 patients infused, with data frozen as of 30 November 2019. Details regarding enrollment are provided in the Supplemental Methods. After lymphodepletion with fludarabine $\left(30 \mathrm{mg} / \mathrm{m}^{2} / \mathrm{d}\right)$ for 4 days and cyclophosphamide $\left(500 \mathrm{mg} / \mathrm{m}^{2} / \mathrm{d}\right)$ for 2 days, patients underwent a single infusion of CARCIK-CD19 cells. The clinical trial followed a 4-dose escalation scheme $\left(1 \times 10^{6}, 3 \times 10^{6}, 7.5 \times 10^{6}\right.$, and $15 \times 10^{6}$ transduced CAR ${ }^{+}$T cells/ $\mathrm{kg}$ ) and was planned to include up to 18 patients treated in cohorts of 3 . The dose assignment for patients who sequentially entered the study was prespecified according to the BOIN (63), a design with desirable statistical properties and performance superior to the traditional 3 +3 design. The threshold for toxicity was set at $30 \%$, and decision rules for the conduction of the trial were guided by the observed rate of dose-limiting toxicity and are depicted in Supplemental Table 6. During the cell-manufacturing period, bridging antileukemic therapy from patient registration to the beginning of lymphodepletion was allowed. Products were thawed at bedside in the pediatric setting and in the Cell Factory in the adult setting.

Assessment of clinical response. The primary endpoint of this study was to define the maximum tolerated dose (MTD) and assess for safety. Toxicity was graded using the National Cancer Institute (NCI) Common Terminology Criteria for Adverse Events (CTCAE) (version 4.03; Supplemental Table 7 and refs. 28, 64).

Key secondary endpoints included the assessment of complete hematologic response (CR), defined as less than $5 \%$ abnormal blasts in $\mathrm{BM}$, less than $1 \%$ circulating blasts, restoration of normal hematopoiesis, and no clinical evidence of extramedullary disease as well as the measurement of CAR T cell persistence in PB and BM. CRi is defined as when all criteria for CR are met, but there are not adequate neutrophils $\left(\leq 1.0 \times 10^{9} / \mathrm{L}\right)$ and platelets $\left(\leq 100 \times 10^{9} / \mathrm{L}\right)$. MRD was assessed by morphology and flow cytometry (limit of detection, $1: 10,000)$ and, in those patients with a known molecular marker, by quantitative PCR (qPCR).

Detection of integrated copy number by qPCR. DNA was extracted from cultured T cells using QIAamp DNA Mini Kit (QIAGEN) according to the manufacturer's instructions. Quantitative real-time PCR was performed on 100 ng of genomic DNA using TaqMan Gene Expression Master Mix (Applied Biosystems) and TaqMan qPCR (RT-PCR System 
A

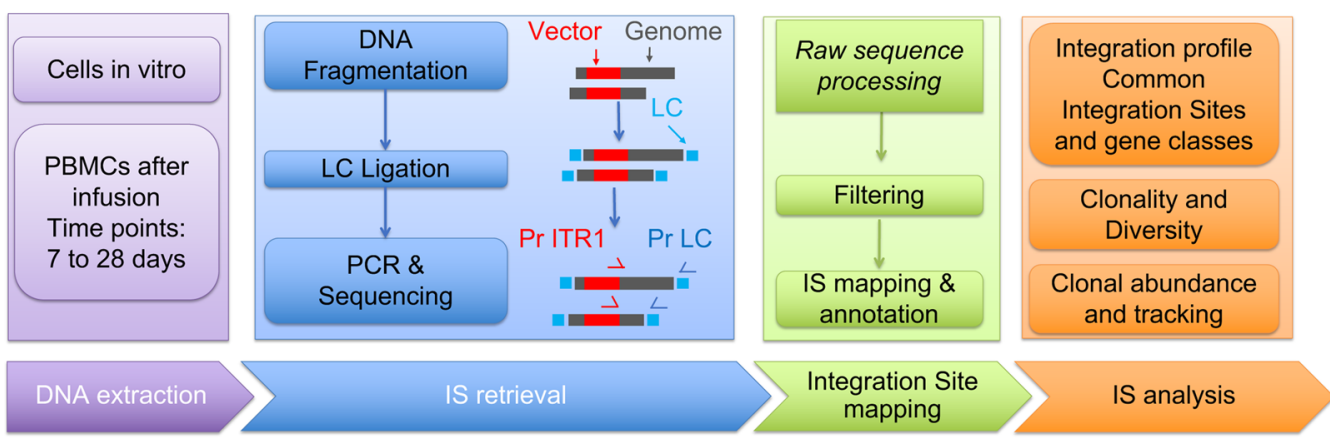

B

Frequency distribuion

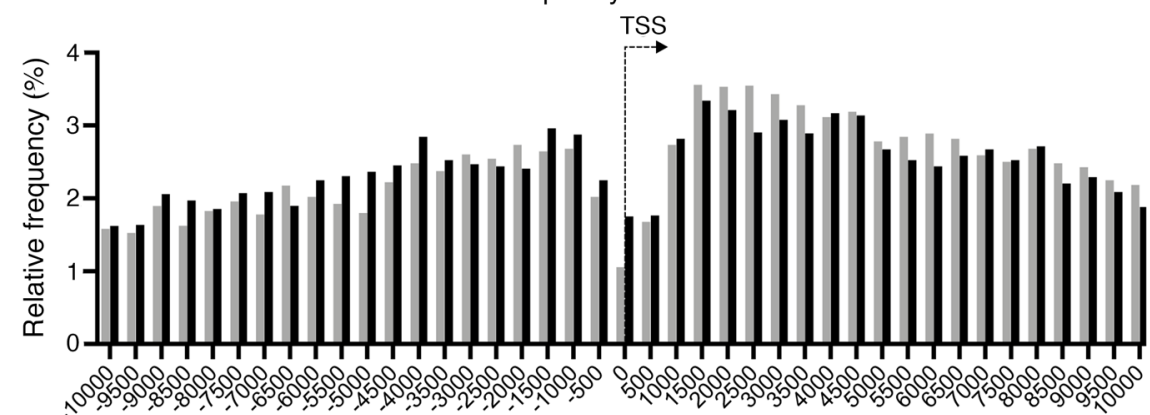

C

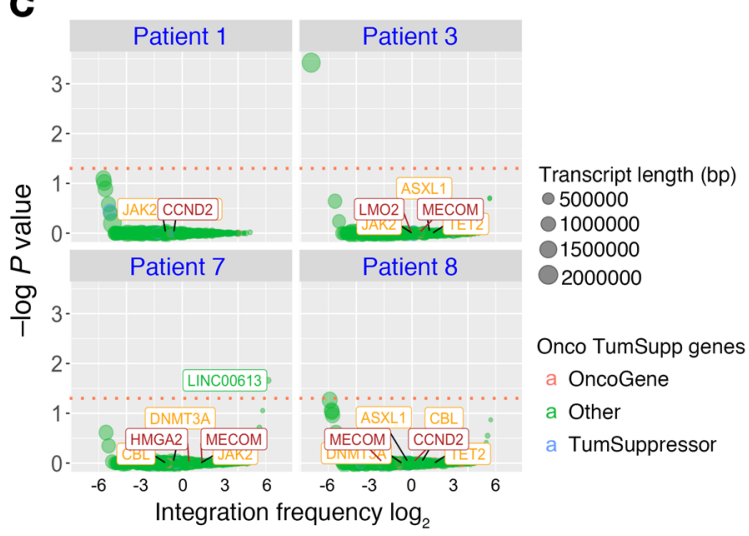

$\mathbf{E}$

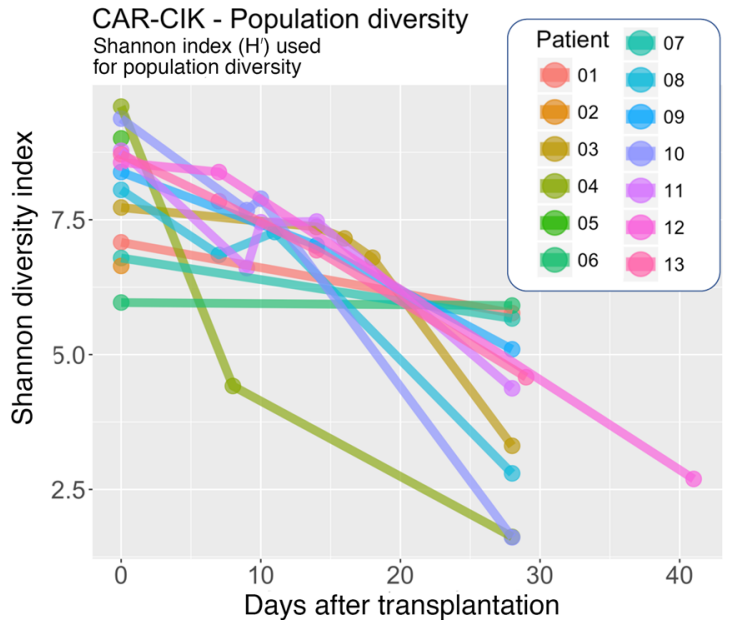

D
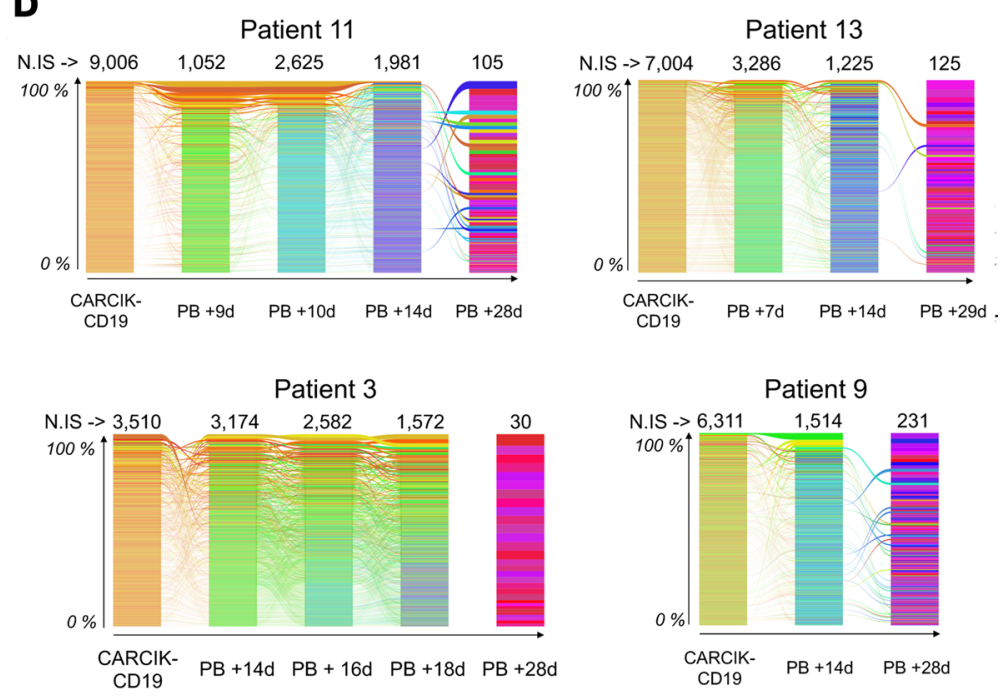
Figure 6. Transposon IS analyses in patient PB and cellular products. (A) Experimental and analytical workflow adopted for IS retrieval and analysis in vitro and in vivo samples. (B) Frequency distribution of SB IS around genes' TSS (interval $\pm 10 \mathrm{~Kb}$ divided in 500 bp bins) in vitro (black bars) and in vivo (gray bars), expressed as percentages relative to the total IS for each data set ( $n=40$ samples). (C) CIS analysis was performed on the IS identified for each patient using the Grubbs test for outliers and volcano plot representation. All genes targeted by IS were tested and plotted with dots of size proportional to the gene length; gene integration frequency normalized by gene length was placed on the $x$ axis, while the $y$ axis shows the $P$ value of the CIS Grubbs test for outliers (- $\log$ base 10 of $P$ value). Tumor suppressor genes are annotated in blue, protooncogene in red, and a generic "other" in green for the remaining genes. Dots with significant $P$ values ( $\alpha$ threshold of 0.05 ) are above the dashed horizontal line and labeled with the closest gene name (RefSeq). Gene ID labels of genes in involved in clonal expansion of genetically modified cells were annotated using red labels, tumor suppressor genes using orange labels, and genes with other functions in blue. (D) For each patient, the clonal abundance of IS represented by at least 2 sequencing reads are represented with a stacked bar plot in which each clone is represented by a different color and the height is proportional to the relative percentage of genomes of the specific IS over the total genomes; each bar represents a specific time point after infusion; ribbons connect tracked clones between 2 consecutive time points. Four out of 11 patients are represented. (E) Shannon diversity $(\mathrm{H})$ index was calculated for the IS data sets obtained from each patient over time $(n=13)$

QuantStudio 3, Applied Biosystems) by comparing hRNaseP qPCR results with VCN qPCR. This method has been developed from previous studies $(24,65,66)$ and validated at Laboratorio Tettamanti according to international guidelines (limit of quantification [LOQ] $<50$ copies/ $\mu \mathrm{g})$. The following primers and probes for the IR/DR sequences were used: Tr-VCN, forward: CTCGTTTTTCAACTACTCCACAAATTTCT; Tr-VCN, reverse: GTGTCATGCACAAAGTAGATGTCCTA; Tr-VCNPR-labeled (FAM) MGB probe, CTGACTTGCCAAAACT.

Pharmacokinetic analysis. The $\mathrm{C}_{\max }$, the time to the maximum observed expansion $\left(\mathrm{T}_{\max }\right)$, and the AUC at 28 days and at the last observation were calculated. AUCs were estimated through the trapezoidal rule.

Assessment of CAR T cell persistence by flow cytometry. $\mathrm{PB}$ and $\mathrm{BM}$ samples were obtained from patients either before CARCIK-CD19 infusion or at multiple time points after infusion. Flow cytometry was performed for identifying $\mathrm{CD} 4^{+}$and $\mathrm{CD} 8^{+} \mathrm{CAR} \mathrm{T}$ cells as well as viable $\mathrm{CD} 45^{+} \mathrm{CD}^{+} \mathrm{CAR}^{+}$cells by incubation with $\mathrm{CD} 19$ his (Thermo Fisher Scientific) and anti-His (Miltenyi Biotec). Absolute CAR T cell counts were determined by correlation of the percentage of positive population to WBC. Details on the antibodies used for immunophenotyping are reported in Supplemental Table 8.

Evaluation of serum cytokines. Serum concentrations of GM-CSF, IFN- $\gamma$, IL-10, IL-12P70, IL-13, IL-15, IL-17, IL-1RA, IL-1 $\alpha$, IL-1 $\beta$, IL-2, IL-3, IL-4, IL-6, IL-7, IL-8, TNF- $\alpha$, and TNF- $\beta$ were evaluated using the MAGPIX System (Millipore, Merck KGaA), combined with MILLIPLEX magnetic bead-based multianalyte panels according to the manufacturer's protocol. The detection limits of the assay were between 0.7 and $8.3 \mathrm{pg} / \mathrm{mL}$, depending on the cytokine.

IS retrieval protocol bioinformatics analysis. Transposon IS were retrieved and sequenced as previously described (67). Amplified PCR products containing the vector/cell genome junctions (IS) were quantified by qPCR in order to compose equimolar sequencing libraries. Additional details regarding the IS retrieval protocol are provided in the Supplemental Methods. Libraries of pooled PCR products were sequenced with the MiSeq Illumina platform. All sequence data obtained in the IS analysis study are available at the NCBI's SRA database (PRJNA643365). Of note, the in vivo samples harvested at the latest time points yield low amounts of PCR products and needed dedicated sequencing runs. Sequencing reads were processed by a dedicated bioinformatics pipeline (VISPA2), as previously described (68). Briefly, paired sequence reads were filtered for quality standards, barcodes identified for sample demultiplexing of the sequence reads, the cellular genomic sequence mapped on the reference Human genome (Human Genome_GRCh37/hg19 Feb. 2019), and the nearest RefSeq gene assigned to each unambiguously mapped IS. CIS analysis was performed using the Grubbs test for outliers, which allows identification of genes in which insertions are significantly enriched with respect to the average gene integration frequency. For the quantification of the abundance of each clone, we adopted an estimation method, SonicLength, based on the analysis of sheared DNA fragments containing the same IS (69). We used custom R scripts to combine data from different samples, filter data set on the known IS, and produce graphical results.

Statistics. Categorical data were summarized by counts and percentages, while continuous data were described with measures of location (i.e., arithmetic or geometric mean, median) and variability (i.e., range, SD, coefficient of variation), as appropriate. The remission rate estimates were reported along with 95\% exact Clopper-Pearson CIs. Statistical analyses were performed with SAS 9.4, except those on GMP manufacture and CAR T persistence, which were performed with GraphPad Prism 5.0. The duration of response was defined as the time from the achievement of CR at 28 days to time of progression. The duration of the follow-up was calculated from the time of the infusion.

Study approval. The phase I/II study entitled "Transposon-manipulated allogeneic CARCIK-CD19 cells in pediatric and adult patients with $\mathrm{r} / \mathrm{r}$ ALL post HSCT (CARCIK)" was performed in accordance with the protocols of the EMA, approved by the Italian Regulatory Central Authorities (AIFA) and by the local ethical committees (Comitato Etico della Provincia di Monza e Brianza), and registered (EudraCT 2017-00900-38 and ClinicalTrials.gov NCT03389035). Written consent was obtained from patients or their guardians in compliance with institutional guidelines and the Helsinki Declaration.

\section{Author contributions}

CFM and G Gaipa contributed equally to this work. CFM acted as the study scientific leader who developed the cellular product, collected and analyzed the data, and wrote the paper. G Gaipa is the head of the GMP facility and of the cytometry facility who led the production of the cellular product and immune monitoring after infusion and contributed to writing the manuscript. The order of the first authors was determined by the respective contributions of CFM and G Gaipa to the work. CFM comes before G Gaipa in the author list because she wrote the manuscript, collected, analyzed the data, led the scientific studies, and developed the cellular product. G Gaipa contributed equally to the study because he led the production of the cellular product and immune monitoring and contributed to writing the manuscript. CFM, G Gaipa, FL, A Balduzzi, EB, GD, A Rambaldi, and A Biondi conceptualized and designed the trial. A Rambaldi and A Biondi were the principal investigators of the study and revised the paper. A Balduzzi and FL were the coinvestigators 
and revised the paper. GD was the clinical study manager. DB, GM, $\mathrm{BC}, \mathrm{CB}, \mathrm{GB}, \mathrm{GF}, \mathrm{SZ}, \mathrm{ST}$, SC, VC, and MQ performed experiments. FB and AC performed IS analysis and analyzed data. GC, EM, and MI designed experiments. G Gritti, SN, SF, and A Rovelli provided medical care for the patients. SG and MGV designed the clinical trial and performed the statistical analyses.

\section{Acknowledgments}

This work was supported by Fondazione AIRC per la Ricerca sul Cancro (AIRC) IG 2017:20564, AIRC/Cancer Research UK (CRUK)/Fundación Científica de la Asociación Española Contra el Cáncer (FC AECC) 22791, Ministero della Salute GR-201602363491, AIRC 5X1000 21147, Fondazione Regionale per la Ricerca Biomedica (FRRB) 2015-0042, FRRB CP2 10/2018, and the Ministero della Salute CAR-T project of the "Rete Oncologica". The authors would like to thank Laurence J. Cooper for providing the SB plasmids; Silvia Rigamonti, Alessio Stevano, and Marianna
Ponzo for assistance in experiments; Riccardo and Donatella Ruschi and the "Amici di Duccio" association, the "Quelli che con Luca" Association, "Comitato Maria Letizia Verga," the Fondazione Benedetta è la vita ONLUS, and "Comitato Stefano Verri" for support; and Markus G. Manz and Renier Myburgh for scientific discussion.

Address correspondence to: Andrea Biondi, Department of Pediatrics, University of Milano-Bicocca, Fondazione MBBM/Ospedale San Gerardo, Via Pergolesi, 33, 20900 Monza, Italy. Phone: 39.039.2333513; Email: abiondi.unimib@gmail.com.

EB's present address is: Clinical R\&D, Celgene, Boudry, Neuchatel, Switzerland.

CFM's present address is: Department of Medical Oncology and Hematology, University Hospital Zurich and University of Zurich, Zurich, Switzerland.
1. Majzner RG, Mackall CL. Clinical lessons learned from the first leg of the CAR T cell journey. Nat Med. 2019;25(9):1341-1355.

2. Borowitz MJ, et al. Prognostic significance of minimal residual disease in high risk B-ALL: a report from Children's Oncology Group study AALL0232. Blood. 2015;126(8):964-971.

3. Bassan R, et al. Improved risk classification for risk-specific therapy based on the molecular study of minimal residual disease (MRD) in adult acute lymphoblastic leukemia (ALL). Blood. 2009;113(18):4153-4162.

4. Gökbuget N, et al. Outcome of relapsed adult lymphoblastic leukemia depends on response to salvage chemotherapy, prognostic factors, and performance of stem cell transplantation. Blood. 2012;120(10):2032-2041.

5. Maude SL, et al. Tisagenlecleucel in children and young adults with B-cell lymphoblastic leukemia. N Engl J Med. 2018;378(5):439-448.

6. Gardner RA, et al. Intent-to-treat leukemia remission by CD19 CAR T cells of defined formulation and dose in children and young adults. Blood. 2017;129(25):3322-3331.

7. Turtle CJ, et al. CD19 CAR-T cells of defined CD4+:CD8+ composition in adult B cell ALL patients. J Clin Invest. 2016;126(6):2123-2138.

8. Park JH, et al. Long-term follow-up of CD19 CAR therapy in acute lymphoblastic leukemia. $N$ Engl J Med. 2018;378(5):449-459.

9. Curran KJ, et al. Toxicity and response after CD19-specific CAR T-cell therapy in pediatric/ young adult relapsed/refractory B-ALL. Blood. 2019;134(26):2361-2368.

10. Ghorashian S, et al. Enhanced CAR T cell expansion and prolonged persistence in pediatric patients with ALL treated with a low-affinity CD19 CAR. Nat Med. 2019;25(9):1408-1414.

11. Frigault MJ, Maus MV. State of the art in CAR T cell therapy for CD19+ B cell malignancies. J Clin Invest. 2020;130(4):1586-1594.

12. Finney OC, et al. CD19 CAR T cell product and disease attributes predict leukemia remission durability. J Clin Invest. 2019;129(5):2123-2132.

13. Fraietta JA, et al. Determinants of response and resistance to CD19 chimeric antigen receptor
(CAR) T cell therapy of chronic lymphocytic leukemia. Nat Med. 2018;24(5):563-571.

14. Ruella M, et al. Induction of resistance to chimeric antigen receptor $\mathrm{T}$ cell therapy by transduction of a single leukemic B cell. Nat Med. 2018;24(10):1499-1503.

15. Schmidt-Wolf IGH, Negrin RS, Kiem HP, Blume KG, Weissman IL. Use of a SCID mouse/human lymphoma model to evaluate cytokine-induced killer cells with potent antitumor cell activity. JExp Med.1991;174(1):139-149.

16. Rambaldi A, Biagi E, Bonini C, Biondi A, Introna M. Cell-based strategies to manage leukemia relapse: efficacy and feasibility of immunotherapy approaches. Leukemia. 2015;29(1):1-10.

17. Introna $\mathrm{M}$, et al. Phase II study of sequential infusion of donor lymphocyte infusion and cytokine-induced killer cells for patients relapsed after allogeneic hematopoietic stem cell transplantation. Biol Blood Marrow Transplant. 2017;23(12):2070-2078.

18. Merker M, et al. Clearance of hematologic malignancies by allogeneic cytokine-induced killer cell or donor lymphocyte infusions. Biol Blood Marrow Transplant. 2019;25(7):1281-1292.

19. Ivics Z, Izsvák Z, Minter A, Hackett PB. Identification of functional domains and evolution of Tc1-like transposable elements. Proc Natl Acad Sci U S A. 1996;93(10):5008-5013.

20. Ivics Z, Hackett PB, Plasterk RH, Izsvák Z. Molecular reconstruction of Sleeping Beauty, a Tc1-like transposon from fish, and its transposition in human cells. Cell. 1997;91(4):501-510.

21. Kebriaei P, et al. Phase I trials using Sleeping Beauty to generate CD19-specific CAR T cells. J Clin Invest. 2016;126(9):3363-3376.

22. Magnani CF, et al. Immunotherapy of acute leukemia by chimeric antigen receptor-modified lymphocytes using an improved Sleeping Beauty transposon platform. Oncotarget. 2016;7(32):51581-51597.

23. Turazzi N, et al. Engineered T cells towards TNFRSF13C (BAFFR): a novel strategy to efficiently target B-cell acute lymphoblastic leukaemia. Br J Haematol. 2018;182(6):939-943.

24. Magnani CF, et al. Preclinical efficacy and safety of CD19CAR cytokine-induced killer cells transfected with Sleeping Beauty transposon for the treatment of acute lymphoblastic leukemia. Hum Gene Ther. 2018;29(5):602-613.

25. Muffly L, et al. Infusion of donor-derived CD8 ${ }^{+}$ memory $\mathrm{T}$ cells for relapse following allogeneic hematopoietic cell transplantation. Blood Adv. 2018;2(6):681-690.

26. Hackett PB, Largaespada DA, Cooper LJN. A transposon and transposase system for human application. Mol Ther. 2010;18(4):674-683.

27. European Commission. EudraLex - Volume 4 Good Manufacturing Practice (GMP) guidelines. https://ec.europa.eu/health/documents/eudralex/vol-4_en. Accessed August 28, 2020.

28. Lee DW, et al. Current concepts in the diagnosis and management of cytokine release syndrome. Blood. 2014;124(2):188-195.

29. Nishimura R, et al. In vivo trafficking and survival of cytokine-induced killer cells resulting in minimal GVHD with retention of antitumor activity. Blood. 2008;112(6):2563-2574.

30. Firouzi S, et al. Development and validation of a new high-throughput method to investigate the clonality of HTLV-1-infected cells based on provirus integration sites. Genome Med. 2014;6(6):46.

31. Levine BL, Miskin J, Wonnacott K, Keir C. Global manufacturing of CAR T cell therapy. Mol Ther Methods Clin Dev. 2017;4:92-101.

32. Schuster SJ, et al. Tisagenlecleucel in adult relapsed or refractory diffuse large B-cell lymphoma. NEngl JMed. 2019;380(1):45-56.

33. Roddie C, O'Reilly M, Dias Alves Pinto J, Vispute K, Lowdell M. Manufacturing chimeric antigen receptor T cells: issues and challenges. Cytotherapy. 2019;21(3):327-340.

34. Kebriaei P, Izsvák Z, Narayanavari SA, Singh $\mathrm{H}$, Ivics Z. Gene therapy with the Sleeping Beauty transposon system. Trends Genet. 2017;33(11):852-870.

35. Mátés L, et al. Molecular evolution of a novel hyperactive Sleeping Beauty transposase enables robust stable gene transfer in vertebrates. $\mathrm{Nat}$ Genet. 2009;41(6):753-761.

36. Clauss J, et al. Efficient non-viral T-cell engi- 
neering by Sleeping Beauty minicircles diminishing DNA toxicity and miRNAs silencing the endogenous T-cell receptors. Hum Gene Ther. 2018;29(5):569-584.

37. Querques I, et al. A highly soluble Sleeping Beauty transposase improves control of gene insertion. Nat Biotechnol. 2019;37(12):1502-1512.

38. Introna $\mathrm{M}$, et al. Repeated infusions of donor-derived cytokine-induced killer cells in patients relapsing after allogeneic stem cell transplantation: a phase I study. Haematologica. 2007;92(7):952-959.

39. Introna $\mathrm{M}$, et al. Rapid and massive expansion of cord blood-derived cytokine-induced killer cells: an innovative proposal for the treatment of leukemia relapse after cord blood transplantation. Bone Marrow Transplant. 2006;38(9):621-627.

40. Provasi E, et al. Editing T cell specificity towards leukemia by zinc finger nucleases and lentiviral gene transfer. Nat Med. 2012;18(5):807-815.

41. Qasim W, et al. Molecular remission of infant B-ALL after infusion of universal TALEN gene-edited CAR T cells. Sci Transl Med. 2017;9(374):eaaj2013.

42. Stadtmauer EA, et al. CRISPR-engineered T cells in patients with refractory cancer. Science. 2020;367(6481):eaba7365.

43. Bailey SR, Maus MV. Gene editing for immune cell therapies. Nat Biotechnol. 2019;37(12):1425-1434.

44. Liu E, et al. Use of CAR-transduced natural killer cells in CD19-positive lymphoid tumors. $\mathrm{N} \mathrm{EnglJ}$ Med. 2020;382(6):545-553.

45. Liu E, et al. Cord blood NK cells engineered to express IL-15 and a CD19-targeted CAR show long-term persistence and potent antitumor activity. Leukemia. 2018;32(2):520-531.

46. Maluski M, et al. Chimeric antigen receptorinduced BCL11B suppression propagates NK-like cell development. JClin Invest. 2019;129(12):5108-5122.

47. Brandjes BD, Davila ML. Adding chimeric antigen receptor-induced killer cells to the medical oncology shelf. J Clin Invest.
2019;129(12):5077-5078.

48. Mueller KT, et al. Clinical pharmacology of tisagenlecleucel in B-cell acute lymphoblastic leukemia. Clin Cancer Res. 2018;24(24):6175-6184.

49. Maude SL, et al. Chimeric antigen receptor $\mathrm{T}$ cells for sustained remissions in leukemia. $N$ Engl JMed. 2014;371(16):1507-1517.

50. Pulè MA, et al. A chimeric T cell antigen receptor that augments cytokine release and supports clonal expansion of primary human T cells. $\mathrm{Mol}$ Ther. 2005;12(5):933-941.

51. Guedan S, et al. Enhancing CAR T cell persistence through ICOS and 4-1BB costimulation. JCI Insight. 2018;3(1):96976.

52. Zhao Z, et al. Structural design of engineered costimulation determines tumor rejection kinetics and persistence of CAR T cells. Cancer Cell. 2015;28(4):415-428.

53. Teachey DT, et al. Identification of predictive biomarkers for cytokine release syndrome after chimeric antigen receptor T-cell therapy for acute lymphoblastic leukemia. Cancer Discov. 2016;6(6):664-679.

54. de Jong J, et al. Chromatin landscapes of retroviral and transposon integration profiles. PLoS Genet. 2014;10(4):e1004250.

55. Cesana D, et al. HIV-1-mediated insertional activation of STAT5B and BACH2 trigger viral reservoir in T regulatory cells. Nat Commun. 2017;8(1):498.

56. Shah NN, et al. Clonal expansion of CAR T cells harboring lentivector integration in the $\mathrm{CBL}$ gene following anti-CD22 CAR T-cell therapy. Blood Adv. 2019;3(15):2317-2322.

57. Fraietta JA, et al. Disruption of TET2 promotes the therapeutic efficacy of CD19-targeted T cells. Nature. 2018;558(7709):307-312.

58. Nobles CL, et al. CD19-targeting CAR T cell immunotherapy outcomes correlate with genomic modification by vector integration. J Clin Invest. 2020;130(2):673-685.

59. Spyridonidis A, et al. Outcomes and prognostic factors of adults with acute lymphoblastic leukemia who relapse after allogeneic hematopoietic cell transplantation. An analysis on behalf of the Acute Leukemia Working Party of EBMT. Leukemia. 2012;26(6):1211-1217.

60. Biagi E, Biondi A, Magnani CF, Tettamanti S, inventors. Improved method for the generation of genetically modified cells. European patent EP3018200A1. July 11, 2014.

61. Challita PM, et al. Multiple modifications in cis elements of the long terminal repeat of retroviral vectors lead to increased expression and decreased DNA methylation in embryonic carcinoma cells. J Virol. $1995 ; 69(2): 748-755$.

62. Geurts AM, et al. Gene transfer into genomes of human cells by the sleeping beauty transposon system. Mol Ther. 2003;8(1):108-117.

63. Yuan Y, Hess KR, Hilsenbeck SG, Gilbert MR. Bayesian optimal interval design: a simple and well-performing design for phase I oncology trials. Clin Cancer Res. 2016;22(17):4291-4301.

64. Lee DW, et al. ASTCT consensus grading for cytokine release syndrome and neurologic toxicity associated with immune effector cells. Biol Blood Marrow Transplant. 2019;25(4):625-638.

65. Kolacsek O, et al. Excision efficiency is not strongly coupled to transgenic rate: cell typedependent transposition efficiency of sleeping beauty and piggyBac DNA transposons. Hum Gene Ther Methods. 2014;25(4):241-252.

66. van der Velden $\mathrm{VH}$, et al. Analysis of minimal residual disease by Ig/TCR gene rearrangements: guidelines for interpretation of real-time quantitative PCR data. Leukemia. 2007;21(4):604-611.

67. Marktel S, et al. Intrabone hematopoietic stem cell gene therapy for adult and pediatric patients affected by transfusion-dependent $ß$-thalassemia. Nat Med. 2019;25(2):234-241.

68. Spinozzi G, et al. VISPA2: a scalable pipeline for high-throughput identification and annotation of vector integration sites. BMC Bioinformatics. 2017;18(1):520.

69. Berry CC, et al. Estimating abundances of retroviral insertion sites from DNA fragment length data. Bioinformatics. 2012;28(6):755-762. 